\title{
Magnetic Resonance Imaging Features of Unusually Dilated Virchow-Robin Spaces
}

\author{
-Two Case Reports-
}

\author{
Masaki KOMIYAMA, Toshihiro YASUI, and Toru IZUMI* \\ Department of Neurosurgery, Osaka City General Hospital, Osaka; *Department of Neurology, \\ Tsukazaki Memorial Hospital, Himeji, Hyogo
}

\begin{abstract}
Two patients presented with unusually dilated Virchow-Robin spaces appearing as cystic lesions of varying size with signal intensity identical to the cerebrospinal fluid on all magnetic resonance pulse sequences. However, fluid-attenuated inversion-recovery (FLAIR) images disclosed small, high intensity foci adjacent to these cystic lesions in one patient. These high intensity foci on FLAIR images may represent chronic ischemic change, which produces the gradual dilation of the Virchow-Robin spaces.
\end{abstract}

Key words: fluid-attenuated inversion-recovery imaging, magnetic resonance imaging,

Virchow-Robin space

\section{Introduction}

Unusually dilated Virchow-Robin spaces mimic ischemic lesions on magnetic resonance (MR) imaging, so the differentiation of dilated Virchow-Robin spaces from ischemic lesions, or any other pathological lesions, is important. Unusually dilated VirchowRobin spaces apparently have a non-pathognomonic nature, ${ }^{1,3-8-10,14)}$ but the pathogenesis and clinical importance are still unclear. Fluid-attenuated inversion-recovery (FLAIR) sequences in MR imaging provide $\mathrm{T}_{2}$-weighted images without artifacts due to cerebrospinal fluid (CSF) by allowing the longitudinal magnetization of the CSF to return to the null level with a long inversion time. ${ }^{7,16]}$ This achieves differentiation of the CSF from ischemic or other pathological lesions. This study describes the MR imaging appearance of two cases of unusually dilated Virchow-Robin spaces on FLAIR images, as well as $\mathrm{T}_{1^{-}}$and $\mathrm{T}_{2}$-weighted spin-echo images.

\section{Case Presentation}

Case 1: A 41-year-old normotensive male developed sudden frontalgia, floating sensation, dysarthria, and weakness and numbness in his left hand. He

Received August 27, 1997; Accepted December 19, 1997 was admitted to our hospital 5 days postictus. His medical history was unremarkable. On admission, he was alert and showed no apparent weakness. However, he had mild sensory disturbance to pain and touch on the left, including the face, but his position sense was normal. He had no cerebellar signs, and laboratory data, including CSF data, was within normal limits.

Computed tomography demonstrated multiple round, low density lesions predominantly in the left deep white matter which were not enhanced by contrast material. $\mathrm{T}_{1}$ - and $\mathrm{T}_{2}$-weighted spin-echo $\mathrm{MR}$ imaging (1.5 $\mathrm{T}$ ) showed multiple lesions with identical signal intensity to the CSF (Fig. 1A, B). The lesions were well-demarcated without mass effect, and were not enhanced by contrast material (Fig. 1C). Cerebral angiography failed to reveal any abnormality. The diagnosis was unusually dilated VirchowRobin spaces with some ischemic change.

Three years after the initial presentation, followup MR imaging examination using fast FLAIR images in addition to $T_{1}$ - and $T_{2}$-weighted spin-echo images was performed. Spin-echo MR imaging showed no changes in the lesions. Fast FLAIR imaging demonstrated low intensity cystic lesions, suggesting dilated Virchow-Robin spaces in addition to small, high intensity foci adjacent to the cystic lesions (Fig. 1D, E). We could not define the lesion responsible for his neurological deficit. There was 

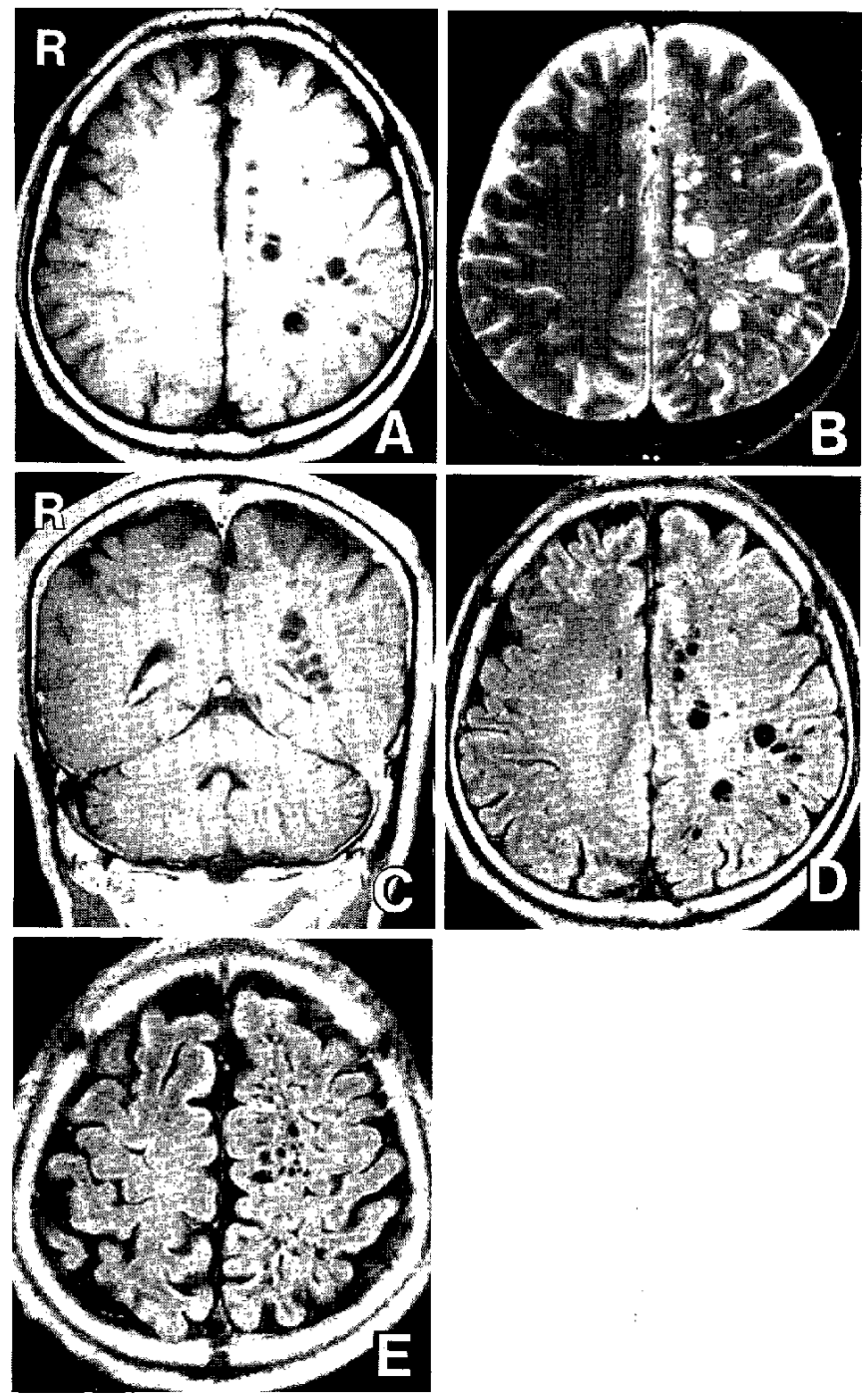

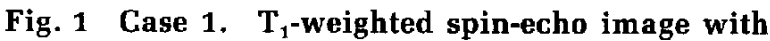
scanning parameters of repetition time 632 msec, echo time $16 \mathrm{msec}$, excitations 2, slice thickness $7 \mathrm{~mm}(632,16,2,7)$ (A) and $T_{2}$ weighted spin-echo image $(2500,80,2,7)$ with a half-Fourier data acquisition (B) showing cystic round lesions of various sizes predominantly in the left centrum semiovale with identical signal intensities to the cerebrospinal fluid (CSF) and no mass effect. $T_{1}$-weighted image $(738,16,2,5)$ with contrast enhancement (C) showing no enhancement within the cystic lesions. The lesions are aligned along the route of the perforating arteries. Fast fluid-attenuated inversion-recovery images $(7500,145,4,7)$ with an inversion time of $1750 \mathrm{msec}$ and an echo train length of $19(D, E)$ disclosing most cystic lesions with low intensity identical to the CSF, and additional small high intensity, not necessarily round lesions adjacent to the low intensity cystic Iesions. no recurrence of the cerebral ischemia during the follow-up period of 3 years.

Case 2: A 59-year-old normotensive female underwent a screening MR imaging examination for posterior neck pain. Her neurological status was normal without remarkable medical history. MR imaging (1.5 T) disclosed an anterior communicating artery aneurysm and multiple cystic lesions in the left, deep white matter near the midline. Both $\mathrm{T}_{1^{-}}$and $\mathrm{T}_{2^{-}}$ weighted spin-echo MR imaging showed these lesions with identical intensity to the CSF (Fig. 2A, B). The lesions were not enhanced by contrast material. In addition, $\mathrm{T}_{2}$-weighted MR imaging showed symmetrical bilateral periventricular hyperintensity. Cerebral angiography revealed only the anterior communicating artery aneurysm. The aneurysm was clipped through the left pterional approach without any sequelae.

Follow-up MR imaging, including fast FLAIR imaging, 2 years later disclosed the cystic lesions with no remarkable changes (Fig. 2C). FLAIR imaging showed the lesions with identical signal intensity to the CSF. The center of some lesions had slightly increased intensity, due to CSF flow artifacts. No high intensity lesions abutting the cystic lesions were observed. During the follow-up period, no neurological events occurred.

\section{Discussion}

Virchow-Robin spaces normally appear as isointensity to the CSF, that is, as low intensity on $\mathrm{T}_{1}$-weighted MR images, and as high intensity on $\mathrm{T}_{2}$-weighted MR images. ${ }^{4,13)}$ However, they sometimes appear as low intensity on $T_{2}$-weighted $M R$ images due to flow voids in the large perforating arteries with relatively less CSF ${ }^{12]}$ MR imaging shows that VirchowRobin spaces occur in three areas: a) the anterior commissure and basal ganglia, along the path of the lenticulostriate arteries; b) the convexity white matter, along the path of the perforating arteries branching from the cortical arteries; and c) the midbrain, along the path of the perforating arteries from the posterior cerebral arteries. ${ }^{4,9,11-13,151}$

Unusually dilated Virchow-Robin spaces typically appear on MR imaging as: a) round, oval, or curvilinear lesions with a well-defined, smooth margin; b) lesions with identical signal intensities to the CSF in any pulse sequences; $c$ ) lesions located along the path of the perforating arteries; d) lesions without mass effects; and e) lesions without contrast enhancement. ${ }^{1,3,8-10,14]}$ However, subtle mass effects are sometimes present. ${ }^{14)}$ Most patients have unilateral lesions in the convexity white matter, predominantly located in the occipital and parietal lobes, but 

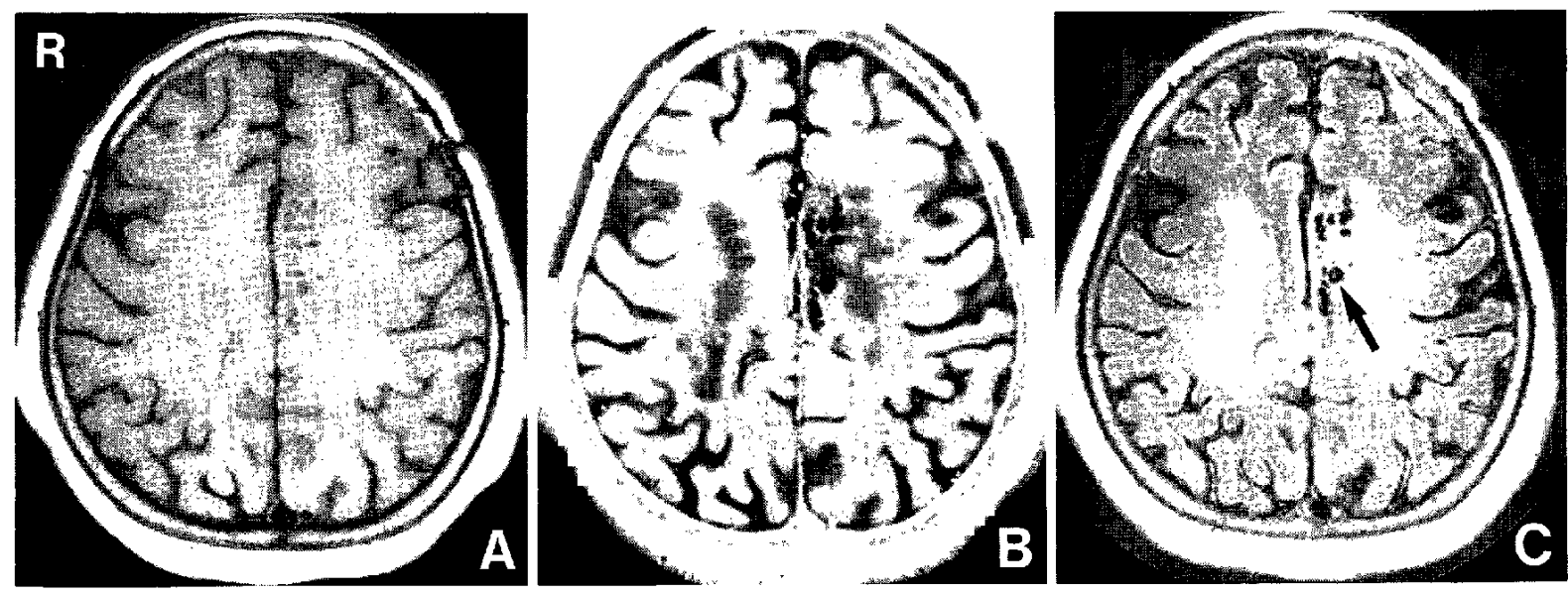

Fig, 2 Case 2. $T_{1}$-weighted spin-echo image $(550,17,2,3)(A), T_{2}$-weighted fast spin-echo image $(2930,110,4,5)$ with an echo train length of $19(B)$, and fast fluid-attenuated inversion-recovery (FLAIR) image $(7500,145,4,5)$ (C) with an inversion time of $1750 \mathrm{msec}$ and an echo train length of 19 showing multiple small cystic lesions in the left centrum semiovale with identical signal intensity to the cerebrospinal fluid (CSF). Periventricular hyperintensity is apparent bilaterally, especially on the FLAIR image. The centers of the cystic lesions on the FLAIR image show slightly higher intensity than the periphery, suggesting CSF flow artifacts (arrow].

some have bilateral lesions., ${ }^{1,3)}$ Unusually dilated Virchow-Robin spaces occur even in the pediatric group. ${ }^{1,15)}$

FLAIR sequences provide $\mathrm{T}_{2}$-weighted, CSFnulled images in which lesions with prolonged $\mathrm{T}_{1}$ and $\mathrm{T}_{2}$ values appear as high intensity lesions. ${ }^{2,7,16]}$ FLAIR images can demonstrate a wide range of brain diseases ${ }^{7}$ and offer advantages for the evaluation of infarction over conventional or fast spinecho images. ${ }^{2,6]}$ Although FLAIR sequences null or markedly reduce the signal from the CSF, rapid CSF flow increases the signal due to incomplete nulling. ${ }^{2,7,16)}$ This phenomenon is observed in the foramen of Monro, in the adjacent frontal horns of the lateral ventricles, and in the posterior fossa. ${ }^{2)}$

FLAIR imaging in our cases showed two patterns of lesions: a) small, medium, or large, but not necessarily round lesions with identical signal intensity to the CSF; and b) small high intensity foci located adjacent to the former. Both types had no mass effect, and no contrast enhancement. The high intensity foci in Case 1 may be due to incomplete nulling of the signals from the CSF. However, the high intensity should be observed at the center of the cystic lesions, as shown in Case 2. Therefore, the high intensity foci at the periphery of the lesions are probably not caused by incomplete nulling of the CSF signals. FLAIR imaging demonstrated chronic infarctions as a mixture of low intensity in the center, suggesting liquefaction, and high intensity on the peripheral rim. ${ }^{2)}$ FLAIR imaging showed acute infarction as high intensity, and chronic infarction which exhibits cystic encephalomalacia as the CSF intensity which is nulled by the FLAIR sequences. ${ }^{6]}$

Unusually dilated Virchow-Robin spaces have a benign nature, supported by examination of biopsy specimens. ${ }^{14)}$ No histopathological examination of lesions was possible in our cases, but possible pathological changes include increased water content, gliosis, and ischemic change. The FLAIR observations suggest that chronic ischemic change due to sclerosis and ectasia of the perforating arteries appearing as high intensity foci became low intensity cystic lesions resulting in dilation of the Virchow-Robin spaces. There was no apparent increase in the number or size of the lesions in our series during 2-3 years, so the progression of the pathological change may be very slow. MR imaging shows infarction with cystic change, brain cyst, and ventricular diverticulum as isointense to the CSF., ${ }^{43}$ Old infarctions with cystic change should be associated with neurological symptoms, ${ }^{14 j}$ but we believe that ischemic change with slowly progressive cyst formation can occur without apparent neurological manifestation. Thus, the clinical significance of dilated or unusually dilated Virchow-Robin spaces remains to be elucidated.

Invasive diagnostic examinations for unusually dilated Virchow-Robin spaces are unnecessary. However, careful follow-up examination using 
FLAIR MR imaging can detect chronological changes in such lesions. Further accumulation of data is necessary to clarify the pathogenesis and clinical importance.

\section{Acknowledgments}

The authors thank Mr. James Sweet, Stanford University School of Law, Stanford, U.S.A., for his assistance in preparation of this manuscript.

\section{References}

1) Afifi AK: Enlarged Virchow-Robin spaces along the medullary perforators in a child with seizures. J Neuroimaging 6: 197-198, 1996

2) Alexander JA, Sheppard S, Davis PC, Salverda P: Adult cerebrovascular disease: role of modified rapid fluid-attenuated inversion-recovery sequences. AJNR Am J Neuroradiol 17: 1507-1513, 1996

3) Bacheschi LA, Magalhāes ACA, Mathias SC: Multiple cystic lesions in white matter without clinical manifestations (Unidentified black holes). Neuroradiology 37 (Suppl): 246-247, 1995

4) Braffman $\mathrm{BH}$, Zimmerman RA, Trojanowski JQ, Gonatas NK, Hickey WF, Schlaepfer WW: Brain MR: Pathologic correlation with gross and histopathology. 1. Lacunar infarction and Virchow-Robin spaces. AJNR Am J Neuroradiol 9: 621-628, 1988

5) Braffman BH, Zimmerman RA, Trojanowski JQ, Gonatas NK, Hickey WF, Schlaepfer WW: Brain MR: Pathologic correlation with gross and histopathology. 2. Hyperintense white-matter foci in the elderly. AJNR Am J Neuroradiol 9: 629-636, 1988

6) Brant-Zawadzki M, Atkinson D, Detrick M, Bradley WG, Scidmore G: Fluid-attenuated inversion recovery (FLAIR) for assessment of cerebral infarction. Initial clinical experience in 50 patients. Stroke 27: 1187-1191, 1996

7) De Coene B, Hajnal JV, Gatehouse P, Longmore DB, White SJ, Oatridge A, Pennock JM, Young IR, Bydder GM: MR of the brain using fluid-attenuated inversion recovery (FLAIR) pulse sequences. AJNR Am J Neu- roradiol 13: 1555-1564, 1992

8) Demaerel P, Wilms G, Baert AL, Bergh VV, Sainte T: Widening of Virchow-Robin spaces. AJNR Am J Neuroradiol 17: 800-801, 1996

9) Elster AD, Richardson DN; Focal high signal on MR scans of the midbrain caused by enlarged perivascular spaces: MR-pathologic correlation. AJNR Am J Neuroradiol 11: 1119-1122, 1990

10) Hasegawa O, Nakamori A, Kubota H, Suzuki $Y$, Kawaguchi Y: Large Virchow-Robin spaces presented by MRI. Shinkei Naika 36: 93-95, 1992

11) Heier LA, Bauer CJ, Schwartz L, Zimmerman RD, Morgello S, Deck MDF: Large Virchow-Robin spaces: MR-clinical correlation. AJNR Am J Neuroradiol 10: 929-936, 1989

12] Hirabuki N, Fujita N, Fujii K, Hashimoto T, Kozuka $\mathrm{T}$ : MR appearance of Virchow-Robin spaces along lenticulostriate arteries: Spin-echo and two-dimensional fast low-angle shot imaging. AJNR Am J Neuroradiol 15: 277-281, 1994

13) Jungreis CA, Kanal E, Hirsch WL, Martinez AJ, Moossy J: Normal perivascular spaces mimicking lacunar infarction: MR imaging. Radiology 169: 101104, 1988

14) Ogawa $T$, Okudera T, Fukusawa $H$, Hashimoto $M$, Inugami A, Fujita $\mathrm{H}$, Hatazawa J, Shimosegawa $\mathrm{E}$, Noguchi K, Uemura K, Nakajima S, Yasui N: Unusual widening of Virchow-Robin spaces: MR appearance. AJNR Am J Neuroradiol 16: 1238-1242, 1995

15) Rollins NK, Deline C, Morriss MC: Prevalence and clinical significance of dilated Virchow-Robin spaces in childhood. Radiology 189: 53-57, 1993

16) Rydberg JN, Hammond CA, Grimm RC, Erickson BJ, Jack CR, Huston J, Riederer SJ: Initial clinical experience in MR imaging of the brain with a fast fluidattenuated inversion-recovery pulse sequence. Radiology 193: 173-180, 1994

Address reprint requests to: M. Komiyama, M.D., Department of Neurosurgery, Osaka City General Hospital, 2-13-22 Miyakojima-Hondouri, Miyakojima-ku, Osaka 534-0021, Japan. 